\title{
A Scoping Review on the Accuracy of Fit of Removable Partial Dentures in a Developing Digital Context
}

This article was published in the following Dove Press journal: Clinical, Cosmetic and Investigational Dentistry

\author{
Noor Al Mortadi ${ }^{1}$ \\ Karem H Alzoubi (D) $^{2}$ \\ Robert Williams ${ }^{3}$ \\ 'Department of Applied Dental Sciences, \\ Faculty of Applied Medical Sciences, \\ Jordan University of Science and \\ Technology, Irbid, Jordan; ${ }^{2}$ Department of \\ Clinical Pharmacy, Faculty of Pharmacy, \\ Jordan University of Science and \\ Technology, Irbid, Jordan; ${ }^{3}$ Cardiff School \\ of Sport and Health Sciences, Cardiff \\ Metropolitan University, Cardiff, UK
}

Purpose: The aim of this paper is to evaluate studies on the quality of fit of alloy removable-partial-dentures (RPDs) produced by computer-aided design/computer-aided manufacturing $(\mathrm{CAD} / \mathrm{CAM})$ and rapid prototyping (RP) technology. This paper offers a comprehensive scoping review of various methods of assessing the quality of fit of RPDs in the context of a developing digital approach to manufacturing and assessment.

Methodology/Approach: A search was made using MEDLINE/PubMed, Scopus, and Science Direct to identify the studies of the accuracy of fit of RPDs.

Findings: Optical and physical examination of dentures "in situ" on the model or in the patient's oral cavity were the most commonly used methods for the assessment of fit of RPDs. Eighteen of the included studies assessed the internal fit between RPDs and oral tissues or models using either polyvinyl silicone materials as filler, calipers, photographs, and microscopes or a combination of these. Two studies used visual assessment. Most studies reported that the fit of RPDs ranged from satisfactory to excellent. However, many of these assessments seemed somewhat subjective and flawed with regard to assessing indentations of the framework into a model or distortion. Two papers used a new method of computer-based superimposition which included a color map of discrepancies to assess the fit of the machineproduced RPDs offering possibilities to quantify the assessment of fit, perhaps leading to a more objective assessment. Despite the limited number of clinical trials, the available evidence was thought to reinforce the claim that the fit of RPDs fabricated digitally was acceptable.

Conclusion: The recent introduction of color maps to compare the differences between the fitting surface and the model is promising. However, a new method for displaying discrepancies shown by color maps is introduced, which could lead to a more quantitative assessment.

Keywords: dental fit, removable partial dentures, digital context, review, accuracy

\section{Introduction}

The fit of removable partial dentures (RPDs) is considered one of the most important features in evaluating RPD success and was identified as the chief complaint wearers. ${ }^{1}$ The misfit (distance between the framework and the reference cast, and/or the distortion and indentations produced by the framework) could be a result of poor laboratory procedures, or a distorted impression, or ridge remodeling after delivery. In fact, misfit is the most commonly reported source of dissatisfaction $(76 \%){ }^{2}$ A well-fitting RPD reduces long-term periodontal or structural
Correspondence: Noor Al Mortadi Department of Applied Dental Sciences, Faculty of Applied Medical Sciences, Jordan University of Science and Technology, P. O. Box (3030), Irbid 22 I I0, Jordan

Tel +962799820380

Email Naalmortadi@just.edu.jo 
damage to abutment teeth. ${ }^{3}$ The fit of alloy RPD is usually evaluated either by the assessment of fit on the definitive cast, or the fit of the RPD intraorally, ${ }^{4,5}$

An RPD can be assessed for fit clinically using a dental mirror and cheeks where the rests of RPDs are properly seated touching the teeth in correct places, and that the intraoral soft tissues are not impinged upon by any part of the RPD, such as the major connectors. ${ }^{2}$ Although these techniques may be considered somewhat informal, nevertheless they are used on an everyday basis to assess whether the RPD is well fitted prior to insertion. However, this informality is often carried over into a research context.

Several new materials and methods of producing RPDs have been developed in recent years. Improvements in polymer-based materials and digital fabrication approaches allow increased biocompatibility, durability, and elasticity, as well as more esthetically agreeable and cost-effective outcomes. ${ }^{6}$

Continuing technological advances in the computeraided design/computer-aided manufacturing (CAD/CAM) systems and the materials may offer improved quality, expanded capabilities, and increased user friendliness of the prosthesis ${ }^{7-9}$ These new technologies are potentially important for patients seeking more rapid, accurate, and functionally efficient prosthetic rehabilitation. ${ }^{7,10}$

Early CAD/CAM systems depended entirely on subtractive methods (ie, milling). Recently, additive methods employing rapid prototyping (RP) have progressed rapidly in several fields of dentistry as they have the potential to overcome the possible drawbacks of subtractive techniques. For example, there could be discrepancies of fit due to the difficulty of milling sharp corners or tight troughs with the smallest milling tool, which maybe too large for the corner to be cut. The RP technologies are also able to produce more complex forms. ${ }^{11}$ However, a recent article, which analyzed the fit of a milling-produced coping on a typical tooth preparation for a single crown compared to the fit of an RP-produced coping, found that either manufacturing system can be used in dental practice. ${ }^{12} \mathrm{~A}$ small and insignificant discrepancy of fit between the two manufacturing methods was found with milling showing a slightly improved fit.

The RP describes the customized production of solid models using 3D computer data. Over the past decade, advances in RP have continued to develop, resulting in the development of new techniques that have been applied to the fabrication of various prostheses. The RP fabrication technologies include Stereo Lithography, ${ }^{6}$ Fused Deposition Modeling (FDM), and, more recently, Selective Laser Sintering (SLS). ${ }^{13}$

The RP techniques have been used to build complex 3D models in medicine since the 1990s. The RP has been recently found successful applications in different dental fields, such as the fabrication of implant surgical guides, frameworks for fixed RPDs, wax patterns for the dental prosthesis, zirconia prosthesis, molds for metal castings, maxillofacial prostheses and complete dentures and RPDs. ${ }^{11,14}$

The use of RP in the manufacture of RPDs allows for the elimination of the waxing, investing and casting steps and thus reduces the potential for inaccuracies, possibly resulting in better quality control in the dental lab. ${ }^{14-17}$ Moreover, the use of RP, may allow for an automatic determination of the insertion axis, and fast identification of retentive areas' deletion. ${ }^{10}$ It may potentially reduce the preparation time for RPDs, ${ }^{10,18}$ require fewer adjustments, ${ }^{10,18}$ and reduce material waste. ${ }^{9}$

\section{Objectives}

A prosthesis is considered to be ill-fitting when 'gaps' are evident between any part of an RPD and the definitive cast or oral tissues, or if indentations into the definitive cast or tissues are produced on the fitting surface of the RPD, or distortion. Inappropriate fit may contribute to discomfort, movement, or damage to the associated tissues, lack of function, and poor aesthetics. Improper fit may also be the primary reason that many RPDs are not worn. An ill-fitting prosthesis can exaggerate other problems caused by the prosthesis. An example that reported typical sites where there was a loss of fit, $76 \%$ of RPD rests did not contact the intended surfaces and thus might not function as intended. ${ }^{19}$

This scoping review seeks to review and analyze relevant literature in the field of interest. Various methods of assessing the quality of fit of RPDs manufactured by digital and conventional techniques are discussed. Developments in color mapping may ultimately offer a better solution for research purposes which will be discussed later in this review.

This review was conducted according to the framework adapted and proposed from the methodology of Joanna Briggs Institute (JBI) Scoping Reviews Manual 2015. ${ }^{20}$

\section{Inclusion Criteria}

The inclusion criteria for selecting articles was established (Table 1). Studies involving a subset of relevant research that discussed laboratory procedures followed by 
Table I Inclusion and Exclusion Criteria for Selected Articles

\begin{tabular}{|l|l|}
\hline Inclusion Criteria & Exclusion Criteria \\
\hline - Peer-reviewed, primary & - Not peer-reviewed, primary \\
research. & research. \\
- Published in English. & - Not written in English \\
- Focusing on fit of removable & - Abstract with no full txt \\
partial dentures in digital & - Literature that did not include \\
(context). & empirical data (letters, editorials, \\
- Published between & news etc.). \\
January 2002 and August 2019 & \\
\hline
\end{tabular}

assessment of fit were considered for inclusion. References of selected articles were also reviewed for possible inclusion in the study. Titles and abstracts of all selected articles were reviewed and upon identification for possible inclusion, the full text of the article was reviewed thoroughly and cross-matched with the predefined inclusion criteria. Further, only research and review articles, and case reports and series were included. Primarily, the first researcher screened titles and abstracts for potential eligibility in the study. To increase reliability, this procedure was carried out in collaboration with the librarian/researcher. Two researchers individually screened a random selection of 1283 titles and abstracts. Any variances were discussed and a consensus was reached. Once the screening process was complete, eligible records were obtained as full texts. The final list of included articles was evaluated and verified by the research team.

The articles used in this review were found in MEDLINE PubMed (National Library of Medicine, Washington, DC), Scopus (Elsevier), and Science Direct using Jordan University of Science and Technology library.

\section{Search Strategy}

The keyword combinations in the title or abstract of publications are shown in Box 1. Articles concerning RPDs fabricated using $\mathrm{CAD} / \mathrm{CAM}$ and RP that were published from 2002 to August/2019 were selected which were written in English. Another material was excluded because most digital developments have occurred since 2002 and the cost and time involved in translating material. The definition of search terms was based on an investigation by Lima et al. ${ }^{21}$ The articles which contained the search terms in any part of the article (including titles, abstracts, or article texts) were screened and the articles discussing fit were reviewed.
Box I Keywords and Subject Headings Used During the Search

\section{Search Terms}

"rapid prototyping" and "removable partial denture", "rapid prototyping" and "removable partial denture" and "framework", "additive manufacturing" and "removable partial denture", "rapid manufacturing" and "removable partial denture", "Accuracy" and "RPD", "dental prosthesis design" and "removable partial denture" and "framework", "CAD/CAM" and "removable partial dentures", "CAD" and "removable partial denture", "RP" and "RPDs", "RP" and "removable dentures", "RP" and "removable prosthesis",

"Digitally designed" and "removable dentures", "Digital removable partial dentures", "Rapid manufacturing" and "removable dentures"

Articles of complete dentures and prototyping implants were also excluded except for one which discussed color mapping. Duplicated articles among different search resources were excluded. Articles discussing the production of RPDs from metal-free materials were also excluded. A data charting form was used to record data extracted from included articles. To fulfill the scoping review question, the researchers considered what exact information should be extracted. Out of the original articles 1283 articles which result from various search resources and references, 50 were read in full, and 20 articles were selected for inclusion in the review (Figure 1). After much consideration, the following data from each article was extracted: study design and purpose, sample method, main findings.

\section{Presentation of the Result}

The studies of RPDs were divided into two categories depending on the method of assessing the fit; Analogue methods and Digital methods. A comparison between the accuracy of RPD fit between the two categories will be presented.

Analogue method category involves non-computer studies which and relying on a filling material to register gaps, microscopy or a digital photography camera to assess fit. However, Digital method category involves the studies that use the technology to produce color map to assess fit.

\section{Analogue Methods of Assessing Fit}

A number of studies discussed below used non-computerbased methods to assess fit such as the use of silicone as a registration material to fill gaps between the fitting surface of a physical RPD and the stone master model, or between an RPD clasp and a tooth to measure gaps 


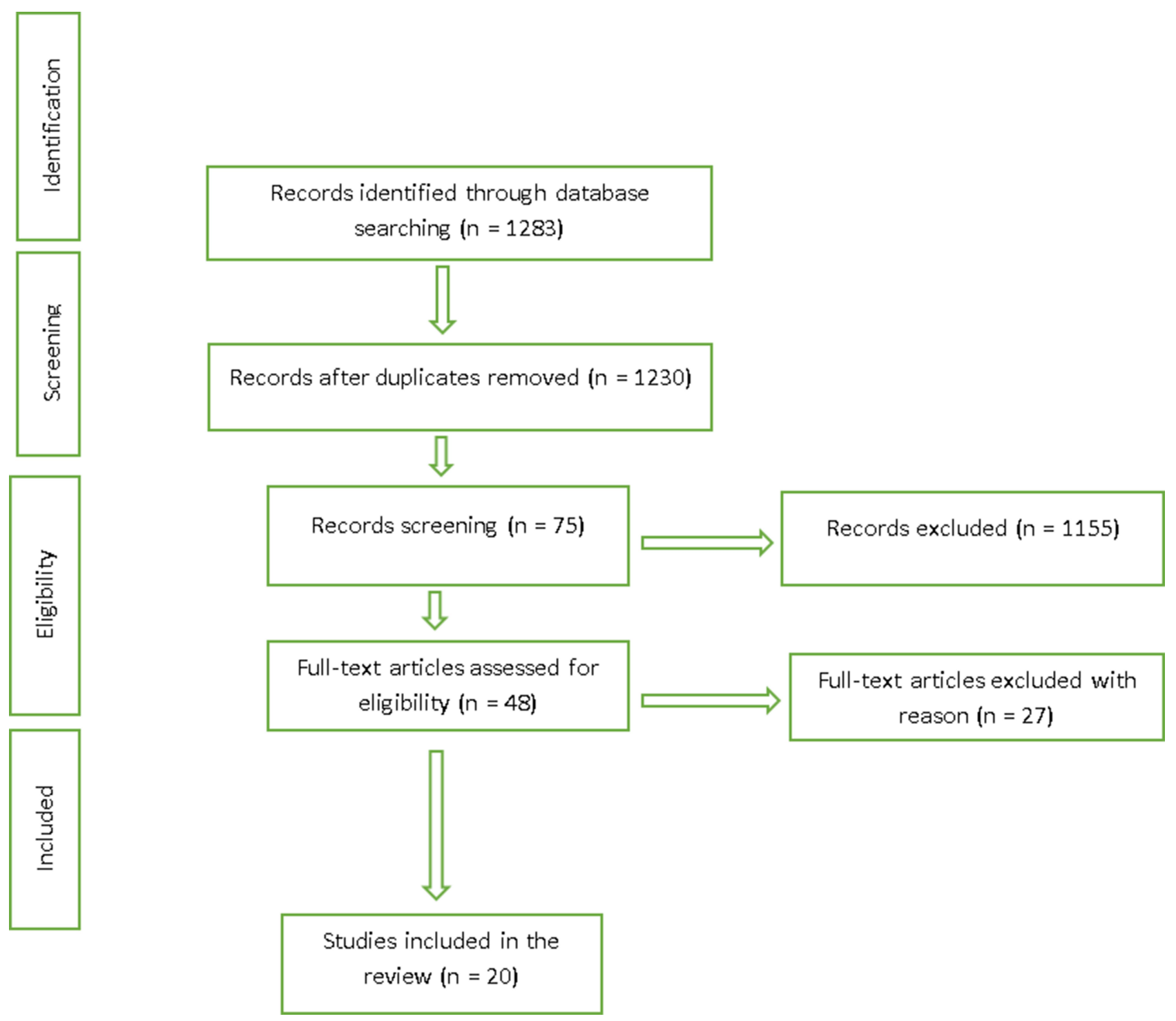

Figure I Identification and selection of studies included.

between these surfaces by measuring the thickness of filling material. ${ }^{22-27}$ Others used silicone to assess the accuracy of fit of a RP-produced clasp on a tooth, and compared it to a conventionally produced clasp on a tooth. $^{26,27}$ A study fabricated a replica using polyvinyl siloxane (PVC) and analyzed the accuracy of the RPD framework. ${ }^{19}$ The accuracy of fit was assessed on the cast by 4 examiners. Gaps between the rests of the 50 clasp assemblies and the rest seats were recorded with PVC and measured in micrometers by a dial caliper. ${ }^{19}$ Measurement of the thickness of a silicone registration material using a caliper is one of the methods most frequently used to determine the accuracy of an RPD. ${ }^{19}$ However, this method has a drawback in that it is difficult to accurately measure the thickness due to the elasticity of the silicone.
The results of the above-mentioned studies which assess fit by relying on a filling material to register gaps are likely to be inaccurate for two important reasons. Firstly, the filler material could potentially distort either on setting or more likely on removal over tight areas, or during measurement. Secondly, this method offers no way of showing whether there are indentations of the dental item into soft tissues or the model or whether such encroachments produce distortions when a restoration or appliance is seated on hard tissues. The same point applies to some studies reviewed above which rely on tactile or visual assessment.

The following articles which use microscopy and a digital photography camera are not subject to the first point above but are still subject to the second criticism. 
A study used light microscopy (VMZM-40; 4H-JENA Engineering $\mathrm{GmbH}$, Germany) at $\times 560$ magnification to determine the accuracy of fit of modified clasps fabricated by 4 different $\mathrm{CAD} / \mathrm{CAM}$ systems and compared their fit to those produced by conventional techniques. ${ }^{28}$ The RPDs were produced using 4 different CAD/CAM techniques which were: indirect RP (wax inject printing combined with lost wax technique [LWT]), direct RP (Selective Laser Melting [SLM]), indirect milling (wax milling with conventional technique), and direct milling (resin milling Polyether ether ketone).$^{28}$ Frameworks were placed on the master model, and the accuracy of each canine clasp (12 clasps per group) was analyzed at 3 vertical and 3 horizontal areas and at 2 vertical and 3 horizontal areas of modified molar clasps. Leaving aside questions regarding whether vertical and horizontal distances are the most meaningful concepts when measuring curved, complex 3D shapes. ${ }^{28}$

A recent study was carried out to compare the fit of 15 RPD frameworks fabricated using the SLM technique to 15 RPDs produced by the conventional method by measuring polyvinyl siloxane (PVC) for both groups using a digital microscope at $50 \mathrm{x}$ in micrometers. ${ }^{24}$ The fit accuracy of digital RPDs fabricated by RP was assessed by measuring silicone registration material with a total of 348 measurements using a stereomicroscope and image program. ${ }^{29}$ Another study used an USB digital intraoral camera at $\times 16.5$ magnification at 3 points on each side to measure visible gaps of 30 RPD frameworks produced by conventional techniques and the light-curing modeling material technique. ${ }^{30}$ Every framework was transferred to an alloy master model to measure gaps between the metal base of the framework and the crest of the alveolar ridge of the model. Authors commented that the accuracy of framework can differ according to the span length of the edentate ridge and material type of framework used for fabrication. ${ }^{30}$ It was reported that the mean value of the short edentulous span was significantly smaller than that of the long edentulous span in the light-curing modeling material technique, whereas the opposite result was obtained in the conventional technique group. ${ }^{30}$ Yet, a different study measured the space under a major connector at the anterior and posterior borders of maxillary palatal strap, using a profile projector with a sensor. ${ }^{30}$ In this study, 24 duplicate for a machine-milled metal cast were used. Twelve were control group while the remaining twelve were included in the test group, which had the provision for anchorage. It was concluded that the accuracy of fit of the palatal major connector was significantly better in the test group than the control group, with $0.1 \%$ level of significance at point $\mathrm{P}$. The accuracy was significantly improved in both groups at point A by $1 \%$ level of confidence. ${ }^{30}$

Visual and tactile examination of RPDs on the cast or in the oral cavity were one of the most used methods to assess the fit of the digitized RPD in several studies. ${ }^{10,17,21,31-37}$ Questions arise relating to the rather informal judgements needed when using visual inspection and movement of frameworks on models or "in situ" to assess whether a dental item is fit for purpose. Where the above studies which use a microscope or digital camera are concerned, questions arise regarding where and in which direction the minimum distance between the complex 3-dimensional structures is taken to be.

\section{Digital Methods of Assessing Fit}

Whilst studies relating to the fit of RPDs have been undertaken for many years, perhaps due to its comparatively recent development, there is little literature exploring the application of the ability of digital technology to create "color maps" to the topic of fit. A color map is a technique where an STL file produced by a 3D scan of, for example, the fitting surface of an RPD is superimposed with an STL file of a scan of the model surface in three dimensions. The "best fit" of these two surfaces is obtained by digital procedures and colors appear indicating the various sizes of gaps or indentations between the RPD and the model. In other words, this technique shows the value of surface discrepancies as a map of colors where each color represents a value of the differences between the two surfaces, for instance in millimeters. Furthermore, these discrepancies are based on, for example, perhaps 600 points of comparison. This offers a huge step change to the number of points it is possible to compare using analogue methods.

Two published papers that use color maps in the assessment of fit of RPDs in the dental literature are discussed here. Negm et al (2019) used metrology software for the color map technique and the misfit (distance between each framework and the reference cast) was measured at 25 standardized points. ${ }^{38}$ In order to assess the overall accuracy, each STL file of the framework was superimposed over the original STL file of the design, and the average deviation was recorded in microns. ${ }^{38}$ In addition, a study by Soltanzadeh et al (2019) used the same software to measure the gap distance between the frameworks and scanned master model at 8 locations. ${ }^{39}$ 
However, the study reports that although both conventional and 3D-printing methods of framework fabrication revealed clinically acceptable adaptation, the conventional cast RPD groups revealed better overall fit and accuracy. ${ }^{39}$ This study raises the question of whether 8 locations are enough to reach a clear conclusion compared to other studies which use hundreds. Improved findings may be possible if more points were selected which is easily possible with color map technology. ${ }^{39}$

\section{Whether the Digital Methods Deliver Better Fit Results Than Analogue Methods for RPDs?}

There are a number of studies that compared the accuracy of fit of RPD between CAD/CAM-produced RPD and conventionally casted RPD. Few of these studies manufactured one or limited number of RPDs to assess the accuracy of fit. Therefore limited novel results are to be expected but a novel critique and new application of existing knowledge has been suggested in these studies.

An "in vitro" study argued that the fitting of 15 RPDs, which were designed using a lab scanner (D800, 3Shape, Copenhagen), and a commercial dental computer-aided design (CAD) software (Dental System 2015, 3Shape, Copenhagen) fabricated from Polyetheretherketone (PEEK) - RPDs were better fitting than those traditionally constructed cast framework RPDs. ${ }^{22}$ In addition, the CAD/ CAM technology caused significantly smaller gap distances in all of the clasp regions, as compared to the conventional technique. ${ }^{26}$ It was also reported that the clasps produced by direct milling (CAD/CAM) showed significantly better fit compared to conventional clasps. ${ }^{27}$ A recent study compared the quality of fit of RP-RPDs and conventionally produced RPDs for 15 selected cases using PVC, concluded that the gap between the rest and relevant rest seat was bigger in RP-RPDs than the conventional castings. ${ }^{40}$ Nevertheless, the authors commented that the RP-produced frameworks were acceptable for clinical application, with satisfactory retention and stability and no undesired rotation. Leaving aside the issue that such gaps are potential traps for plaque, etc., the study made every effort to maximize the number of cases studied. Fifteen cases are still perhaps insufficient to allow firm conclusions to be drawn and further studies are needed to underpin this initial finding. In addition, a study used PVC as a registration material to compare the fit of SLM produced - RPD frameworks and conventionally produced
RPDs when fitted to models in an "in vitro" study. ${ }^{41}$ It was shown that the average gap of the RP frameworks was larger than those of conventional RPDs in three cases while no difference was found in one case. ${ }^{41}$ Thus, this study goes some way to confirm the findings of the study discussed immediately above. However, a study reports that although both conventional and 3D-printing methods of framework fabrication revealed clinically acceptable adaptation, the conventional cast RPD groups revealed better overall fit and accuracy. ${ }^{39}$ The interesting findings for the above-reviewed articles so far suggesting that milling compares more favorably to conventional casting techniques than printing. However, it has been claimed that RPDs produced by RP techniques showed distinct fitting irregularities.

\section{Discussion}

This review has highlighted the results of studies comparing digital methods of manufacture of RPDs with the conventional technique (Table 2). In doing so, it has developed a strong critique of methods used to assess of fit. Digital RPD manufacture is still at an early stage. The number of steps required in digital procedures is reduced over conventional ones, which suggests a potential improvement is possible, as some of the above studies confirm. It is reasonable to expect that eventually digital methods can produce sufficient accuracy. Furthermore, it may be that the ability to shrink or enlarge a digital dental item by minute amounts in CAD either as a whole or in specific areas could eventually lead to greater comfort.

Whilst most authors suggest digital and conventional techniques to produce acceptable frameworks, this conclusion is largely based on informal judgements. For example, gaps observed between rests and rest seats have been reported but have been informally judged to be acceptable by the researchers involved. Such statements would hold a greater acceptance if a consensus could be agreed regarding what spaces and other aspects of fit were acceptable.

A further degree of rigor would perhaps be achieved if the comparison of findings could be based on a more uniform approach. Currently, comparison of various studies is difficult due to the enormous variation in choice of cases. For example, bounded saddles or free end saddles (distal extensions) of various lengths have been used. Several studies evaluating the fit of RPDs used maxillary models, ${ }^{2,18,29,42}$ whilst other studies used mandibular models. ${ }^{6,11,22,25,30,39}$ Different classes and modifications 


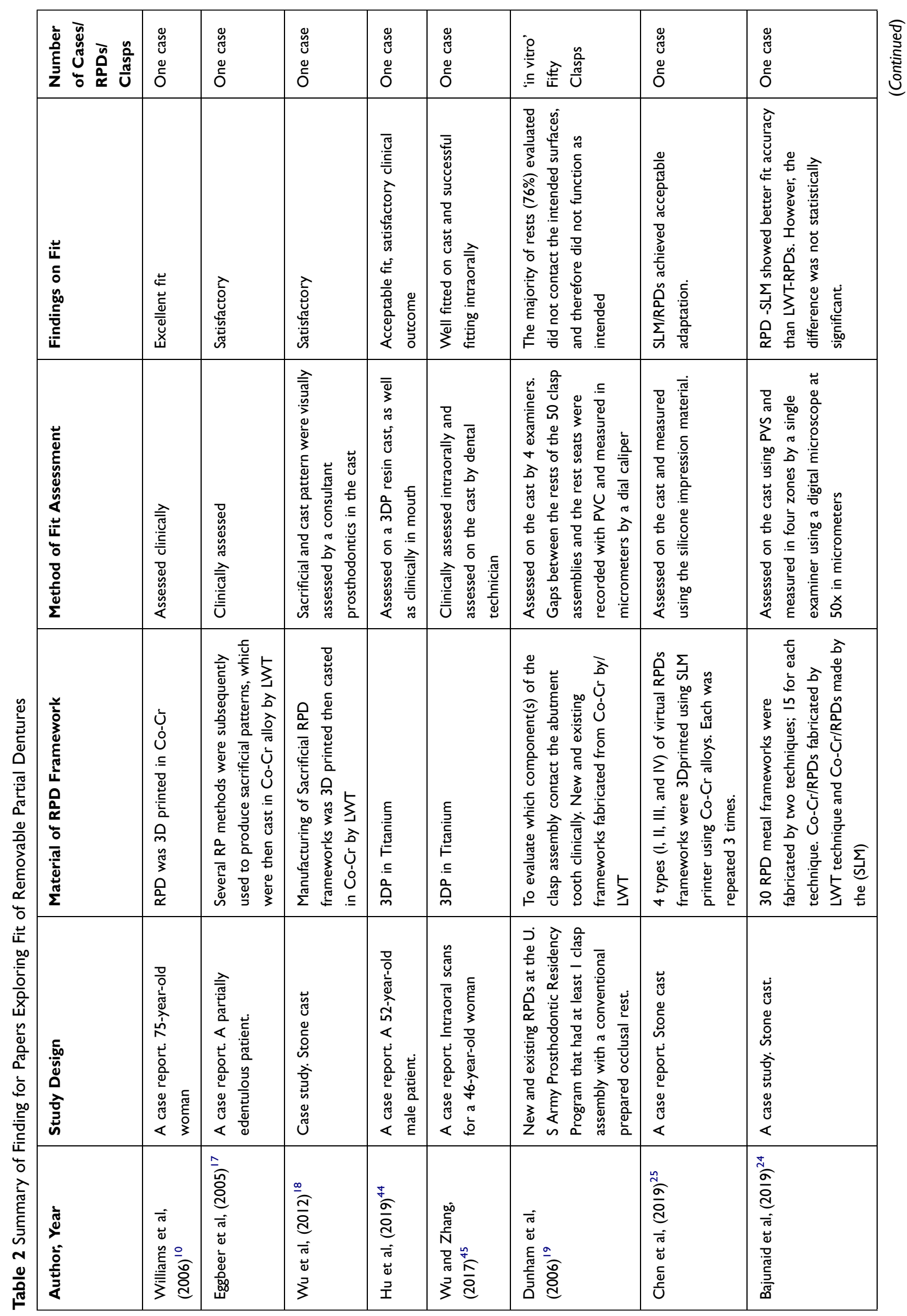




\begin{tabular}{|c|c|c|c|c|c|c|c|}
\hline 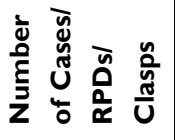 & 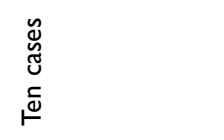 & 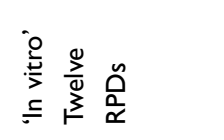 & 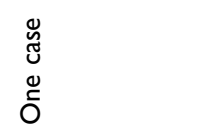 & 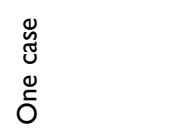 & 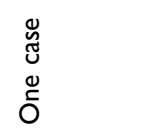 & 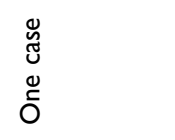 & 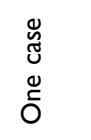 \\
\hline 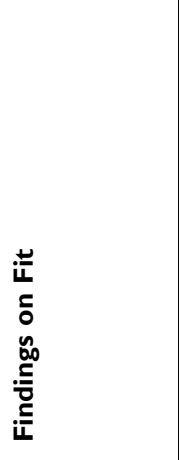 & 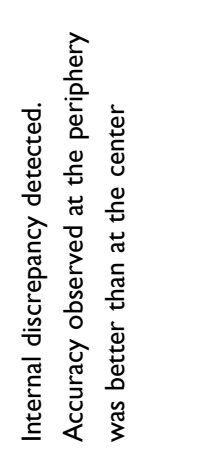 & 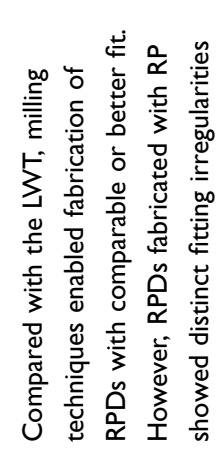 & 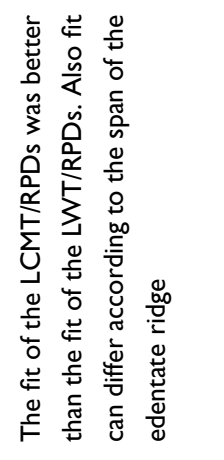 & 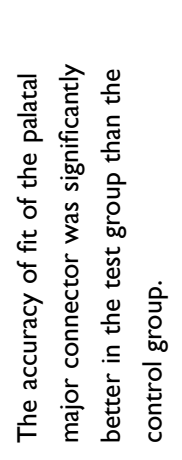 & 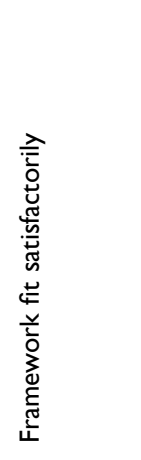 & 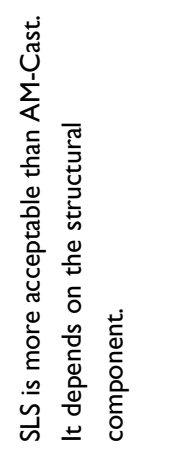 & 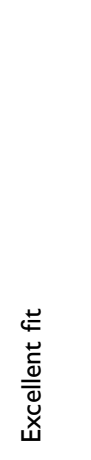 \\
\hline 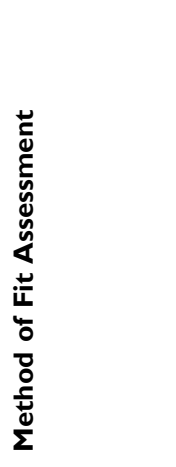 & 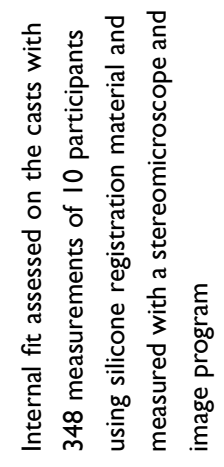 & 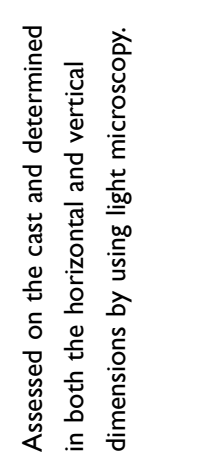 & 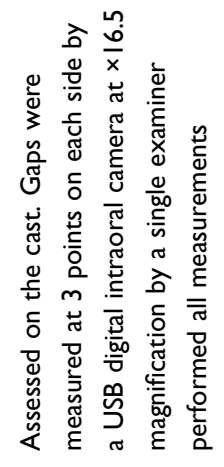 & 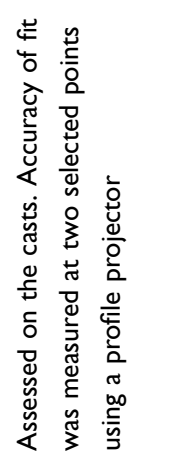 & 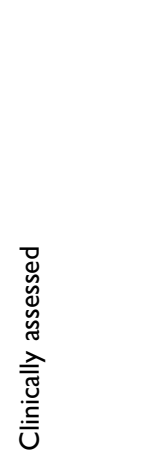 & 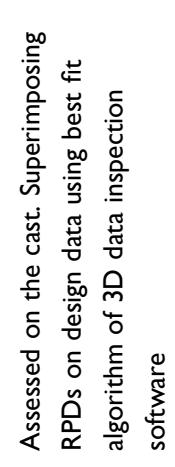 & 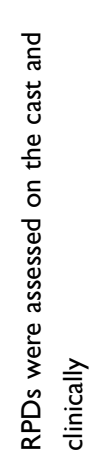 \\
\hline 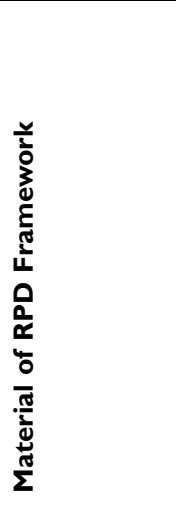 & 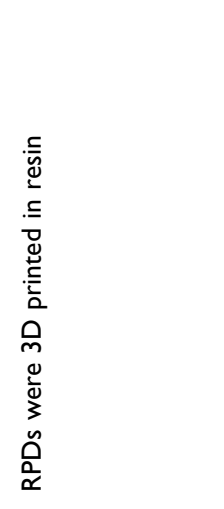 & 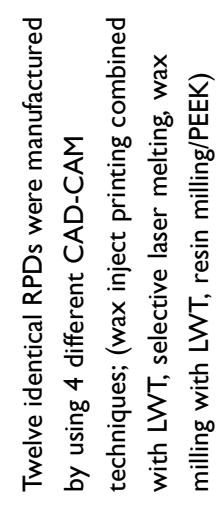 & 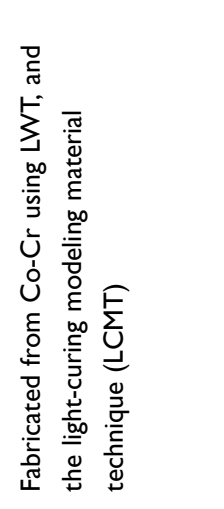 & 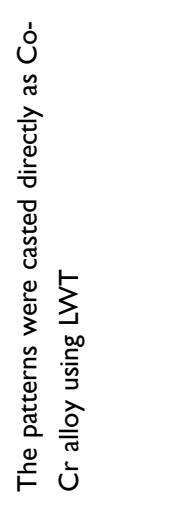 & 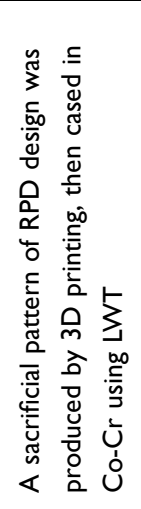 & 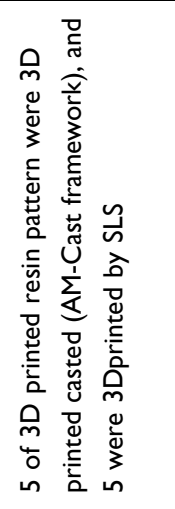 & 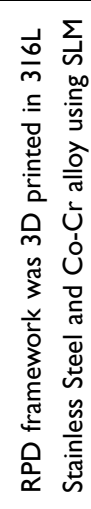 \\
\hline 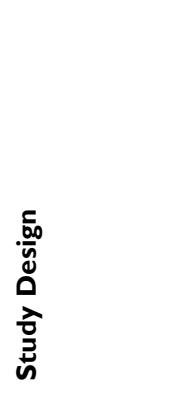 & 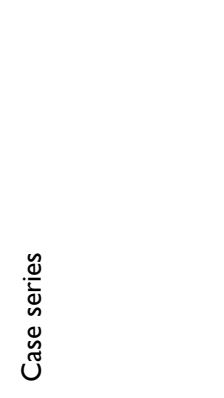 & 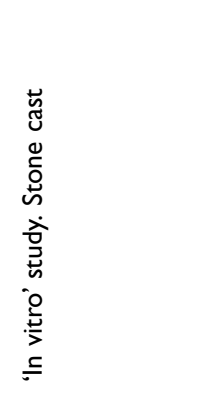 & 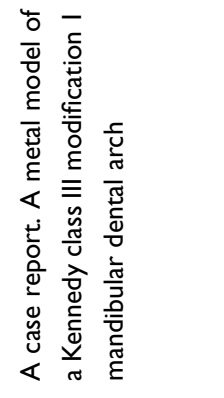 & 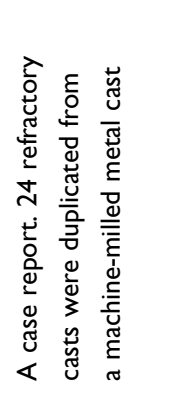 & 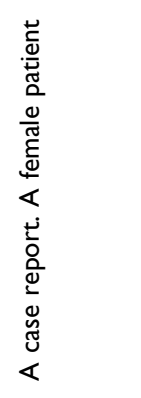 & 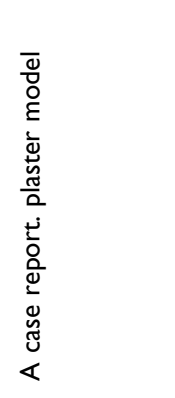 & 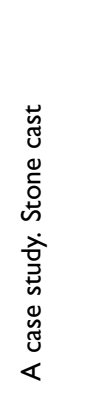 \\
\hline 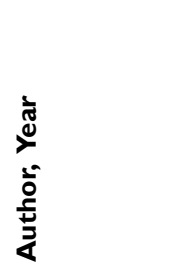 & 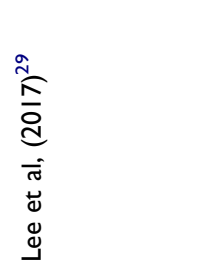 & 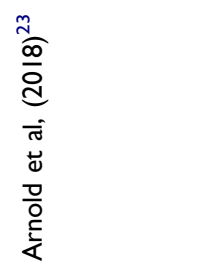 & 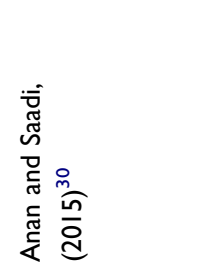 & 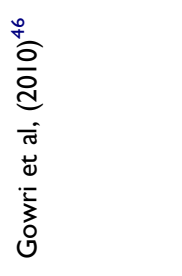 & 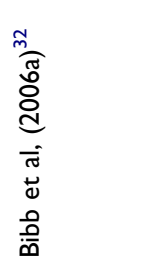 & 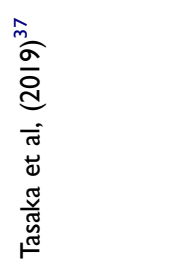 & 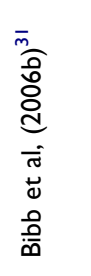 \\
\hline
\end{tabular}




\begin{tabular}{|c|c|c|c|c|}
\hline 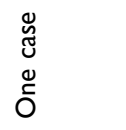 & 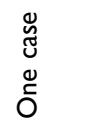 & 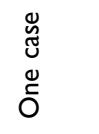 & 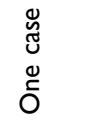 & 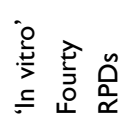 \\
\hline 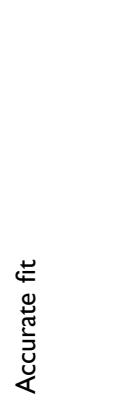 & 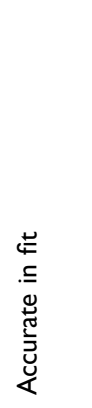 & 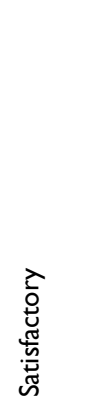 & 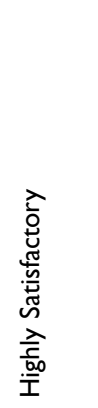 & 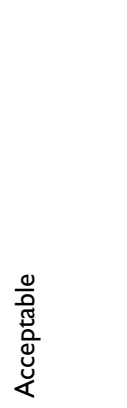 \\
\hline 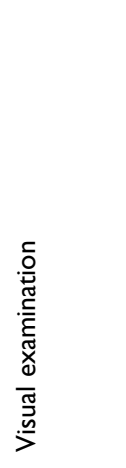 & 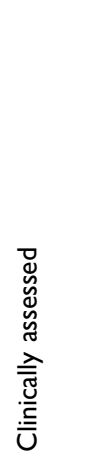 & 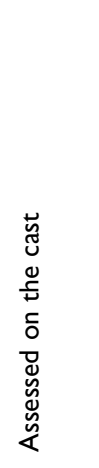 & 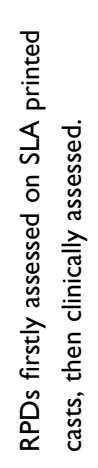 & 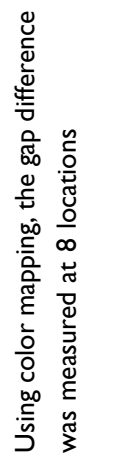 \\
\hline 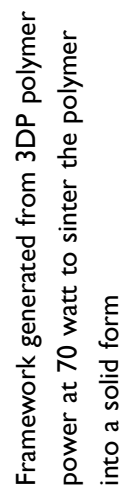 & 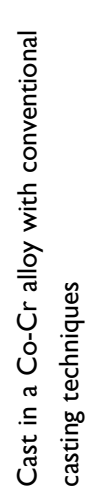 & 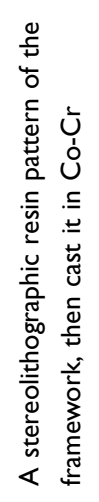 & 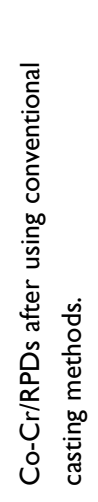 & 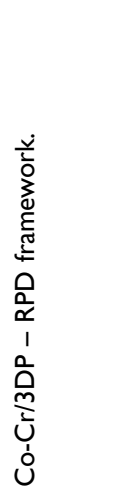 \\
\hline 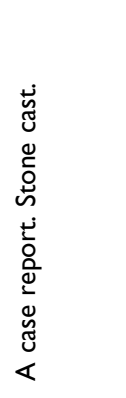 & 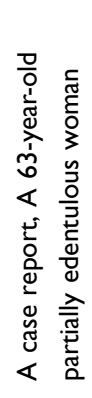 & 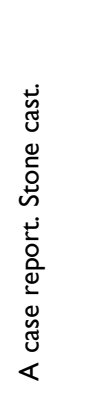 & 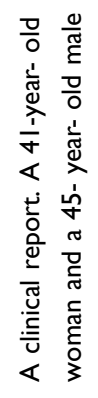 & 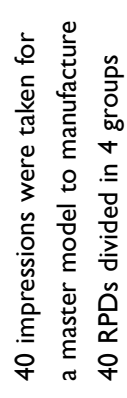 \\
\hline 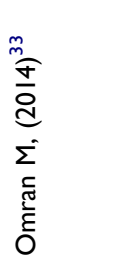 & 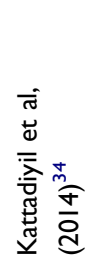 & 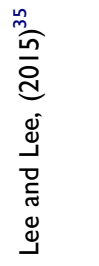 & 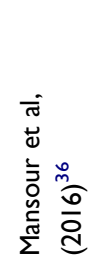 & 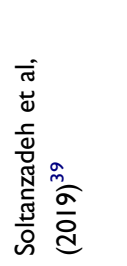 \\
\hline
\end{tabular}

of Kennedy RPDs were also evident in the studies. For instance, a study used a metal model of a Kennedy class III modification 1 with two edentulous spaces of different spans, short and long. ${ }^{30}$ Others used a Kennedy class III modification I. The material type of the models was also different among the studies. ${ }^{39}$

Color maps offer enormous possibilities for the study of fit in dentistry. A color map from an unpublished thesis reproduced here (Figure 2). ${ }^{43}$ Figure 2 shows an image representing a color map, or "color plot" of the fit between the Co-Cr cast and a master cast. Whilst it does not illustrate an RPD but rather an edentulous palatal plate, it provides a good illustration of the use of such maps. The degree of variation between the master model and the casting is represented by colors. In this example, violet represents the master model, blue represents an area which would encroach into a physical model (a negative value), and green represents areas which fit and orange represents an area which would produce a gap between the casting and a physical model. In addition, there are various shades representing measurements in millimeters between the two extremes. For example, violet represents -0.5 to $-0.1 \mathrm{~mm}$, blue represents -0.1 to $-0.05 \mathrm{~mm}$, green represents -0.05 to $0.05 \mathrm{~mm}$, orange represents 0.05 to $0.1 \mathrm{~mm}$ and pink represents 0.5 to $0.25 \mathrm{~mm}$.

However, a method that develops a quantitative assessment of the color map is described below and has not previously appeared in the published dental literature. The method suggests many advantages which will be

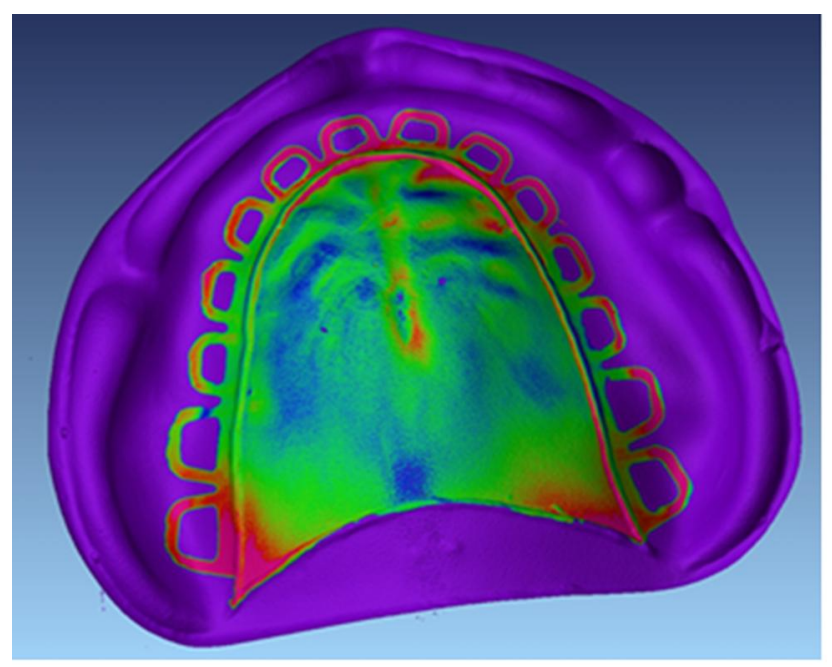

Figure 2 A color plot (Scale $\pm \mathrm{I}$ ) of the fit between the CoCr cast and a master cast. Note: Courtesy of McDowell, M., A comparison of accuracy between BEGO, Siladent, and an Empirical sprueing methodology. - Can computerised radiographic technology assist in identifying most nominal accurate $\mathrm{CoCr}$ casting? [Dissertation], Cardiff Metropolitan University, 2014. 
pointed out. Rather than simply looking, a carefully looking at a vast array of different colors, a suggested approach is to produce a bar chart with very narrow bars. Figure 3 shows such a chart that may typically be generated from the map shown in Figure 2 by software engineering.

In Figure 3 the color of each bar represents the degree of fit or misfit measured in square millimeters across the whole of the fitting surface. There are approximately 150 bars representing an even spread over the fitting surface. The vertical axis shows how many square millimeters there are of each measurement represented by each color. The same or similar colors are then grouped together in the chart. This method is developed dentally in an unpublished thesis. ${ }^{43}$ Each of the 150 (approximately) bars represents a square millimeter of each color, which in turn represents a value of fit $(0.0)$ or misfit and are evenly spread over the fitting surface. The exact fit represented in the histogram is presented at the value of 0.0 shown in green. Hence for the color map used in the present illustration, it can be seen that the majority of points show green, ie, 0.0 (a good fit) and many show a minus value to $0.1 \mathrm{~mm}$. There is a smaller percentage of plus values (or gaps).

However, even a chart such as the one in the illustration does not allow an easy quantification and cannot be read quantitatively by eye because there are so many bars and the colors tend to shade into each other. Yet it does indicate, for example in the figures shown, that there is a greater area of green than blue and a smaller area of red. Perhaps this would not be so easily seen from a visual study of color maps alone. However, from such a chart, a step forward in quantification is easily achieved since it should be possible for software to calculate, as a percentage of the overall area of the fitting surface, where there is fit $(0.0)$, where there are minus values to say, 0.0 to 0.01 square $\mathrm{mm}$, where there are plus values up to say, 0.0 to 0.01 square $\mathrm{mm}$, and so on. Such percentage values would facilitate comparisons of overall fit between studies.

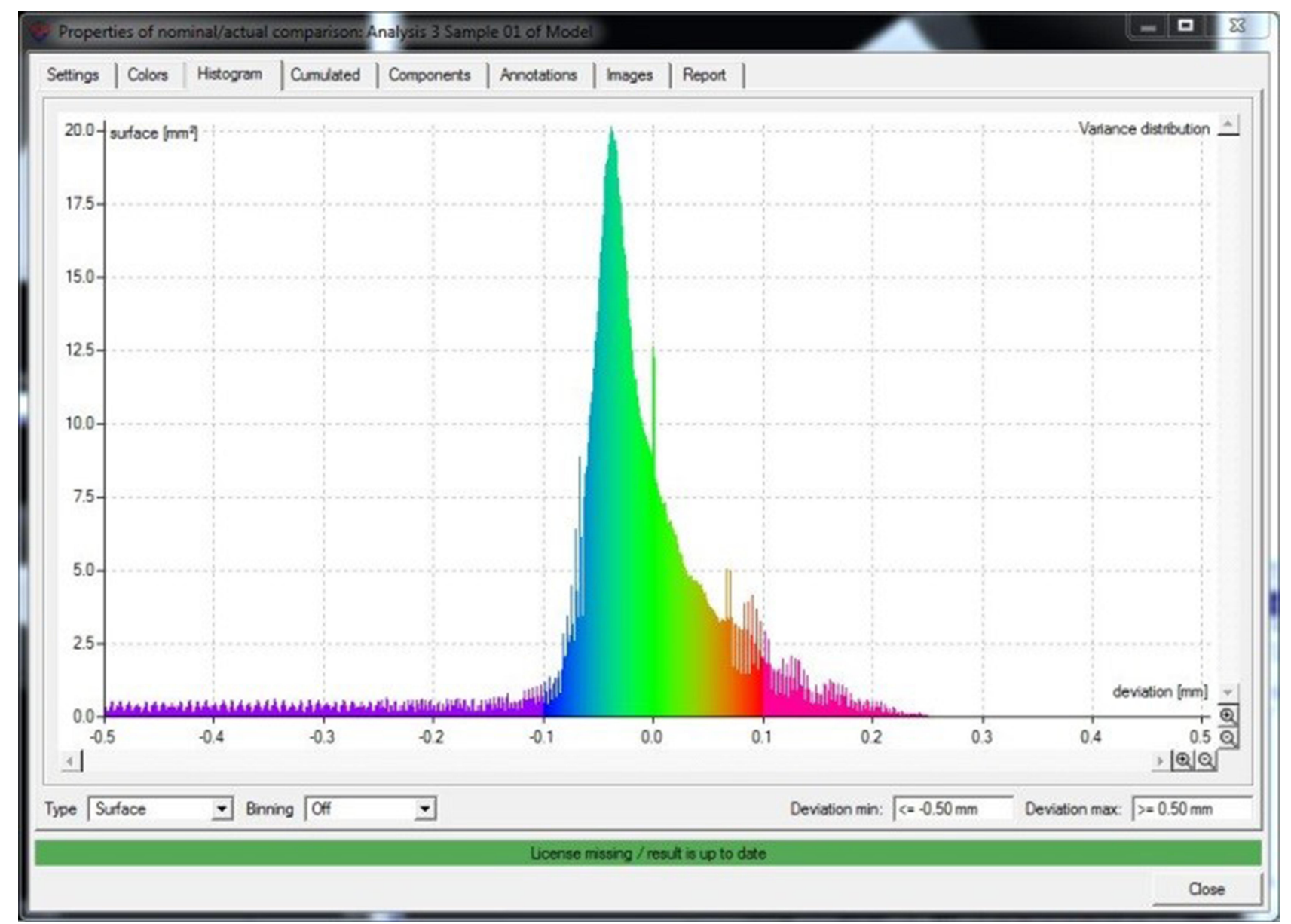

Figure 3 Histograms of nominal fit and misfit.

Note: Courtesy of McDowell, M., A comparison of accuracy between BEGO, Siladent, and an Empirical sprueing methodology. - Can computerised radiographic technology assist in identifying most nominal accurate CoCr casting? [Dissertation], Cardiff Metropolitan University, 2014. 


\section{Conclusion}

In such a context, a standardized approach along the lines of using test procedures that have been prescribed in standards such as for ISOs or the American Standards would help in allowing meaningful comparisons of future studies and give credence to claims regarding what is "an acceptable fit". Finally, a possible method of using color maps, histograms generated from these and percentages of accuracy of fit derived from digital superimposition, has been suggested which again would facilitate quantification and meaningful comparison of studies. This method can replace all other forms of assessment as it does have many advantages. We agree fit and accuracy are the final arbiter, which is why we felt it was vital to include the digital method of assessment of these characteristics.

\section{Acknowledgments}

The authors would like to acknowledge Mr. Michael McDowell for agreeing to the use of parts of his unpublished thesis. The support of Mr. Abdel Salam Ibdah at Jordan University of Science and Technology library is appreciated.

\section{Disclosure}

The authors report no conflicts of interest for this work.

\section{References}

1. Yeung A, Lo E, Clark R, Chow T. Usage and status of cobalt-chromium removable partial dentures 5-6 years after placement. J Oral Rehabil. 2002;29(2):127-132. doi:10.1046/j.13652842.2002.00846.x

2. Frank R, Brudvik J, Leroux B, Milgrom P, Hawkins N. Relationship between the standards of removable partial denture construction, clinical acceptability, and patient satisfaction. J Prosthet Dent. 2000;83 (5):521-527. doi:10.1016/S0022-3913(00)70008-4

3. Mamoun J. The path of placement of a removable partial denture: A microscope based approach to survey and design. $J A d v$ Prosthodont. 2015;7(1):76-84. doi:10.4047/jap.2015.7.1.76

4. Wong MT, Calverley MJ, Nagy W. Removable partial denture framework try-in. J Prosthet Dent. 1993;69(4):363-368. doi:10.1016/00223913(93)90181-M

5. Young L. Try-in of the removable partial denture framework. J Prosthet Dent. 1981;46:579-580.

6. Harb I, Abdel-Khalek E, Hegazy S. CAD/CAM Constructed Poly(etheretherketone) (PEEK) Framework of Kennedy Class I Removable Partial Denture: A Clinical Report. J Prosthodontics. 2018;28.

7. Azari A, Nikzad S. The evolution of rapid prototyping in dentistry: A review. Rapid Prototyping J. 2009;15(3):216-225. doi:10.1108/1355 2540910961946

8. Rekow E. CAD/CAM in dentistry: a historical perspective and view of the future. J Canadian Dental Assoc. 1992;58(283):287-288.

9. Quality control of dental bridges and removable prostheses manufactured using phenix systems equipment. 2012.
10. Williams RJ, Bibb R, Eggbeer D, Collis J. Use of CAD/CAM technology to fabricate a removable partial denture framework. J Prosthet Dent. 2006;96(2):96-99. doi:10.1016/j.prosdent.2006.05. 029

11. Torabi K, Farjood E, Hamedani S. Rapid Prototyping Technologies and their Applications in Prosthodontics, a Review of Literature. J Dentistry. 2015;16:1-9.

12. Zuskova L, Mortadi N, Williams R, Alzoubi K, Khabour O. Comparison of Overall Fit of Milled and Laser-Sintered CAD/CAM Crown Copings. Int J Dent. 2019;2019:1-5. doi:10.1155/2019/ 7310175

13. Begum Z, Chheda P. Rapid Prototyping - When Virtual meets Reality. Int J Comput Dent. 2014;17:297-306.

14. Williams R, Bibb R, Rafik T. A technique for fabricating patterns for removable partial denture frameworks using digitized casts and electronic surveying. J Prosthet Dent. 2004;91(1):85-88. doi:10.1016/j. prosdent.2003.10.002

15. Budak I, Kosec B, Sokovic M. Application of contemporary engineering techniques and technologies in the field of dental prosthetics. $J$ Ach Materials Manufacturing Eng. 2012;54:233-241.

16. Chen G, Zeng X, Wang Z, Guan K, Peng C. Fabrication of Removable Partial Denture Framework by Selective Laser Melting. Adv Materials Res. 2011;317-319:174-178. doi:10.4028/www.scientific.net/AMR.317-319.174

17. Eggbeer D, Bibb R, Williams W. The computer-aided design and rapid prototyping fabrication of removable partial denture frameworks. Proceedings of the Institution of Mechanical Engineers, Part H: Journal of Engineering in Medicine. 2005;219 (3):195-202. doi:10.1243/095441105X9372

18. Wu J, Wang X, Zhao X, Zhang C, Gao B. A study on the fabrication method of removable partial denture framework by computer-aided design and rapid prototyping. Rapid Prototyping J. 2012;2:18.

19. Dunham D, Brudvik J, Morris W, Plummer K, Cameron S. A clinical investigation of the fit of removable partial dental prosthesis clasp assemblies. J Prosthet Dent. 2006;95(4):323-326. doi:10.1016/j. prosdent.2006.02.001

20. Institute TJB. Joanna Briggs Institute Reviewers' Manual: 2015 Edition/Supplement. The Joanna Briggs Institute; 2015.

21. Lima J, Anami L, Araujo R, Pavanelli C. Removable Partial Dentures: use of Rapid Prototyping. J Prosthodontics. 2014;1:23.

22. Ye H, Li X, Wang G, et al. A Novel Computer-Aided Design/ Computer-Assisted Manufacture Method for One-Piece Removable Partial Denture and Evaluation of Fit. Int J Prosthodont. 2018;1:31.

23. Arnold C, Hey J, Schweyen R, Setz J. Accuracy of CAD-CAMfabricated removable partial dentures. J Prosthet Dent. 2017;2:119.

24. Bajunaid S, Altwaim B, Alhassan M, Alammari R. The Fit Accuracy of Removable Partial Denture Metal Frameworks Using Conventional and 3D Printed Techniques: an In Vitro Study. $J$ Contemp Dent Pract. 2019;20(4):476-481. doi:10.5005/jpjournals-10024-2542

25. Chen H, Li H, Zhao Y, Zhang X, Wang Y, Lyu P. Adaptation of removable partial denture frameworks fabricated by selective laser melting. J Prosthet Dent. 2019;122(3):316-324.

26. Ozawa D, Suzuki Y, Kawamura N, Ohkubo C. Fabrication of crown restoration retrofitting to existing clasps using CAD/CAM: fitness accuracy and retentive force. J Prosthodont Res. 2015;59.

27. Torii M, Nakata T, Takahashi K, Kawamura N, Shimpo H, Ohkubo C. Fitness and retentive force of cobalt-chromium alloy clasps fabricated with repeated laser sintering and milling. J Prosthodont Res. 2018;1:62.

28. Arnold C, Hey J, Schweyen R, Setz JM. Accuracy of CAD-CAMfabricated removable partial dentures. J Prosthet Dent. 2018;119 (4):586-592. doi:10.1016/j.prosdent.2017.04.017

29. Lee J-W, Park J-M, Park E-J, Heo S-J, Koak J-Y, Kim S-K. Accuracy of a digital removable partial denture fabricated by casting a rapid prototyped pattern: A clinical study. J Prosthet Dent. 2017;1:118. 
30. Anan MTM, Al-Saadi M. Fit accuracy of metal partial removable dental prosthesis frameworks fabricated by traditional or light curing modeling material technique: an in vitro study. Saudi Dental J. $2015 ; 29$.

31. Bibb R, Eggbeer D, Williams R. Rapid manufacture of removable partial denture frameworks. Rapid Prototyping J. 2006;12(2):95-99. doi:10.1108/13552540610652438

32. Bibb R, Eggbeer D, Williams R, Woodward A. Trial fitting of a removable partial denture framework made using computer-aided design and rapid prototyping techniques. Proceedings of the Institution of Mechanical Engineers, Part H: Journal of Engineering in Medicine. 2006;220(7):793-797. doi:10.1243/0954 4119JEIM62

33. Omran M. Novel 3D Modeling Technique of Removable Partial Denture Framework Manufactured by 3D Printing Technology. Int J Adv Res. 2014;2:686-694.

34. Kattadiyil M, Mursic Z, AlRumaih H, Goodacre C. Intraoral scanning of hard and soft tissues for partial removable dental prosthesis fabrication. J Prosthet Dent. 2014;1:112.

35. Lee J-H, Lee C-H. A stereolithographic resin pattern for evaluating the framework, altered cast partial removable dental prosthesis impression, and maxillomandibular relationship record in a single appointment. J Prosthet Dent. 2015;114.

36. Mansour M, Sanchez E, Machado C. The Use of Digital Impressions to Fabricate Tooth-Supported Partial Removable Dental Prostheses: A Clinical Report. J Prosthodontics. 2015;25.

37. Tasaka A, Shimizu T, Kato Y, et al. Accuracy of removable partial denture framework fabricated by casting with a 3D printed pattern and selective laser sintering. J Prosthodont Res. 2019;64.

38. Negm EE, Aboutaleb FA, Alam-Eldein AM. Virtual Evaluation of the Accuracy of Fit and Trueness in Maxillary Poly(etheretherketone) Removable Partial Denture Frameworks Fabricated by Direct and Indirect CAD/CAM Techniques. J Prosthodontics. 2019;28(7):8 04-810. doi:10.1111/jopr. 13075
39. Soltanzadeh P, Suprono M, Kattadiyil M, Goodacre C, An GW. In Vitro Investigation of Accuracy and Fit of Conventional and CAD/ CAM Removable Partial Denture Frameworks. J Prosthodontics. 2018;5:28.

40. Phoenix RD, Cagna DR, DeFreest CF. Quintessence Publishing Co. Vol. 3. China: Stewart's Clinical Removable Partial Prosthodontics; 2003:95-337.

41. Chen H, Li H, Zhao Y, Zhang X, Wang Y, Lyu P. Adaptation of removable partial denture frameworks fabricated by selective laser melting. J Prosthet Dent. 2019;122(3):316-324. doi:10.1016/j.prosdent. 2018.11.010

42. Negm E, Aboutaleb F, Virtual A-EA. Evaluation of the Accuracy of Fit and Trueness in Maxillary Poly(etheretherketone) Removable Partial Denture Frameworks Fabricated by Direct and Indirect CAD/CAM Techniques. J Prosthodontics. 2019;28.

43. McDowell M. "A comparison of accuracy between BEGO. In: Siladent." Cardiff Metropolitan University (unpublished thesis: and an Empirical sprueing methodology. - Can computerised radiographic technology assist in identifying most nominal accurate $\mathrm{CoCr}$ casting?; 2014.

44. Hu F, Pei Z, Wen Y. Using Intraoral Scanning Technology for Three-Dimensional Printing of Kennedy Class I Removable Partial Denture Metal Framework: A Clinical Report: 3D Printing Metal Framework. J Prosthodontics. 2017;28.

45. Wu J, Li Y, Zhang Y. Use of intraoral scanning and 3-dimensional printing in the fabrication of a removable partial denture for a patient with limited mouth opening. J Am Dental Assoc. 2017;1:148.

46. Gowri V, Patil N, Nadiger R, Guttal S. Effect of Anchorage on the Accuracy of Fit in Removable Partial Denture Framework. J Prosthodontics. 2010;19(5):387-390. doi:10.1111/j.1532-849X.20 10.00594.x
Clinical, Cosmetic and Investigational Dentistry

\section{Publish your work in this journal}

Clinical, Cosmetic and Investigational Dentistry is an international, peer-reviewed, open access, online journal focusing on the latest clinical and experimental research in dentistry with specific emphasis on cosmetic interventions. Innovative developments in dental materials, techniques and devices that improve outcomes and patient

\section{Dovepress}

satisfaction and preference will be highlighted. The manuscrip management system is completely online and includes a very quick and fair peer-review system, which is all easy to use. Visit http://www.dovepress.com/testimonials.php to read real quotes from published authors.

Submit your manuscript here: https://www.dovepress.com/clinical-cosmetic-and-investigational-dentistry-journal 